\title{
EDITORIAL
}

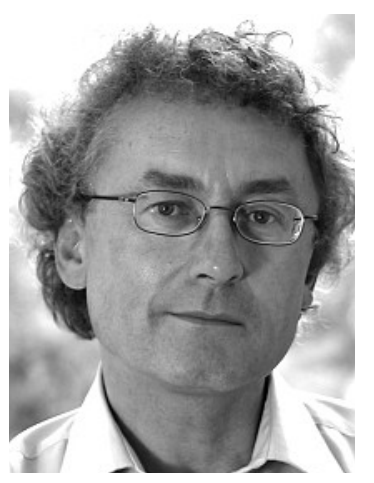

\section{Medicinal Inorganic Chemistry}

Metals play a crucial role in many life-related processes. The corresponding field of research 'Bioinorganic Chemistry' has been intensively investigated by numerous groups worldwide for many decades. The perception that metals can impact on medicine as well is only about 30 years old and is essentially based on the discovery of cisplatin $\left[\mathrm{Pt}\left(\mathrm{NH}_{3}\right)_{2} \mathrm{Cl}_{2}\right]$, the prototype of many metalbased drugs. The impact of this coordination compound for medicinal purposes laid the fundament for what is nowadays called 'Medicinal Inorganic Chemistry'. More recently, the field expanded to 'Medicinal Organometallic Chemistry' as a part of Bioorganometallic Chemistry in general. Although the success of cisplatin could not yet be surpassed, the search for novel metal-containing drugs is pursued at many universities, research institutes and companies. Some novel metal-based drugs are in clinical trials or even shortly before being introduced onto the market. The general interest is also mirrored by different cooperated projects initiated on a European level such as the COST Action D39, 'Metal-based Drug Design and Action' and others more focused on biology or imaging. Since the discovery of cisplatin, research in Medicinal Inorganic Chemistry has substantially diversified. Elements such as ruthenium, gold, rhenium, titanium, iron and others have entered the field. Research is no longer restricted to coordination compounds; organometallic complexes have also proven to be major players in the field thanks to their unique properties.

Medicinal Inorganic Chemistry is a strongly interdisciplinary field. It encompasses traditional inorganic and organic chemistry for complex syntheses and preparations of tailor-made ligands, (bio)physical chemistry for the study of kinetic and thermodynamic properties or interaction of complex-bearing pharmacophores with the (macromolecular) targets, biological chemistry for vector-drug conjugations or structure-activity relationships and biology or medicine for in vitro and in vivo testing of the corresponding compounds. Although interdisciplinary, only fundamental understanding of each of these scientific building blocks will lead to new metal-based drugs. Contributing relevant results out of one of these fields requires broad knowledge and understanding of the others, hence, a very attractive opportunity especially for young researchers.

Owing to the widespread and ongoing research, it is only possible to present a small selection of articles. Nevertheless, we think that this special issue mirrors the dynamic research and gives a good collection of relevant contributions from the frontier of science in Medicinal Inorganic Chemistry. Topics covered range from radioimaging with technetium, cancer therapy in general with ruthenium and breast cancer in particular with iron to developments in platinum chemistry. Biological chemistry is represented by the derivatization strategies of targeting molecules such as peptides with different organometallic moieties. We hope that this special issue of CHIMIA will encourage students to read more about the topic and act as an incentive for them to step into a field of highly dynamic and challenging research.

Roger Alberto

Institute of Inorganic Chemistry

University of Zurich

The Editorial Board of CHIMIA expresses its warmest thanks to the coordinating guest editor Professor Roger Alberto for his efforts in the planning and realization of the present issue on 'Metals in Medicine'. 